\title{
Utilisation des mesures de bruit pour la détermination du photocourant primaire dans les photodiodes à avalanche $\mathbf{N}^{+} \mathbf{P} \pi \mathbf{P}^{+}$au silicium (*)
}

\author{
R. Alabedra, C. Maille, D. Ratsira et G. Lecoy \\ Centre d'Etudes d'Electronique des Solides $(* *)$ \\ Université des Sciences et Techniques du Languedoc, 34060 Montpellier Cedex, France
}

(Reçu le 4 février 1980, révisé le 21 mars 1980, accepté le 1er avril 1980)

\begin{abstract}
Résumé. - L'utilisation d'un photodétecteur nécessite la connaissance de sa puissance équivalente de bruit qui est une fonction du facteur de multiplication des porteurs de charges. Dans une photodiode au Si du type $\mathrm{N}^{+} \mathbf{P} \pi \mathrm{P}^{+}$, l'extension de la zone de charge d'espace et l'ionisation par impact des porteurs entraînent simultanément une augmentation du photocourant initial. L'objet de cet article est de dissocier les deux effets, le premier ayant pour conséquence l'augmentation du photocourant primaire qui sera alors multiplié pour donner le courant résultant. Les mesures de bruit de fond permettent d'atteindre le photocourant non multiplié. Les auteurs montrent dans le cas particulier de ces dispositifs que l'augmentation du photocourant primaire due à l'extension de la charge d'espace peut atteindre un facteur 2. (La non-connaissance de cette valeur entraînant naturellement d'importantes erreurs sur la détermination de la puissance équivalente de bruit de la photodiode en régime de fonctionnement.)
\end{abstract}

\begin{abstract}
The use of a photodetector requires a satisfactory knowledge about the noise equivalent power which is a function of the multiplication of the charge carriers. In a silicon diode of the type $\mathrm{N}^{+} \mathrm{P} \pi \mathrm{P}^{+}$, the extension of the space charge and the impact ionisation together cause an increase in the initial photocurrent. It is the purpose of this paper to make a distinction between these two effects. The former increases the primary photocurrent whereas the latter multiplies it. Noise measurements are used to mark this transition. The authors show in particular that the increase of the primary photocurrent due to the extended space charge region can attain a factor of two (if this value is unknown, the errors in the determination of the noise equivalent power of the photodiode can be substantial).
\end{abstract}

\section{Liste des symboles :}

$a \quad$ : Coefficient d'absorption optique.

$A \quad$ : Surface de la jonction.

$D_{\mathrm{n}}, D_{\mathrm{p}}$ : Coefficient de diffusion des électrons et des trous.

$\varepsilon(x) \quad$ : Valeur du champ en un point $x$ de la zone de charge d'espace.

$\varepsilon_{\max } \quad$ : Valeur maximale du champ électrique à la jonction métallurgique.

$G(x) \quad$ : Taux de génération de paires' électron-trou à une distance $x$.

I : Courant électrique total traversant une jonction.

$I_{0} \quad$ : Courant électrique primaire (non multiplié).

$I_{\mathrm{N}_{0}}, I_{\mathrm{P}_{0}}$ : Photocourant électrique primaire d'électrons et de trous injectés de part et d'autre de la zone de multiplication.

(*) Travail supporté par la Convention CNET no 79 9B 265 BCW/ LAY.

(**) Associé au C.N.R.S.
$I_{\mathrm{W}_{0}} \quad$ : Photocourant électrique primaire des porteurs générés à l'intérieur de la zone de multiplication.

$I_{\mathrm{n}}, I_{\mathrm{p}}$ : Courant électrique d'électrons et de trous.

$J \quad$ : Densité de courant.

$k \quad$ : Rapport $\beta / \alpha$.

$L_{\mathrm{n}}, L_{\mathrm{p}}$ : Longueur de diffusion des porteurs minoritaires : électrons et trous respectivement.

$M \quad$ : Coefficient de multiplication.

$n_{\mathrm{i}} \quad$ : Densité de porteurs intrinsèques.

$N_{\mathrm{d}}, N_{\mathrm{a}}$ : Densité d'impuretés donneurs et accepteurs respectivement.

$N \quad$ : Densité d'impuretés dans la zone $\pi$.

$N_{\mathrm{P}}^{\pi} \quad$ : Densité d'impuretés dans la zone P.

$P \quad$ : Puissance lumineuse.

$\Delta n \quad$ : Densité d'électrons dans la zone $\pi$.

$\Delta p \quad$ : Densité de trous dans la zone $\mathrm{N}^{+}$.

$q \quad$ : Charge de l'électron.

$R \quad$ : Coefficient de réflexion optique d'une surface.

$R_{\mathrm{p}} \quad:$ Profondeur de l'implantation ionique.

$\Delta R_{\mathrm{p}} \quad$ : Ecart autour de $R_{\mathrm{p}}$. 
$s \quad$ : Vitesse de recombinaison en surface.

$S \quad$ : Dose d'implantation ionique.

$S_{\mathrm{i}}(f)$ : Densité spectrale des fluctuations de courant de bruit.

$V \quad$ : Tension appliquée.

$V(x)$ : Potentiel électrique à une distance $x$.

$W \quad$ : Longueur de la zone de charge d'espace.

$W_{\mathrm{A}}$ : Longueur de la zone d'avalanche $\left(\simeq R_{\mathrm{p}}\right)$.

$x_{\mathbf{j}} \quad$ : Profondeur de la jonction.

$x_{\mathrm{c}} \quad$ : Longueur de la zone $\pi$.

$\alpha, \beta$ : Coefficient d'ionisation de l'électron et du trou.

$\varphi_{1} \quad$ : Flux lumineux arrivant sur une surface.

$\varphi_{0} \quad$ : Flux lumineux transmis à l'intérieur du cristal.

$\lambda \quad$ : Longueur d'onde.

$\mu_{\mathrm{n}}, \mu_{\mathrm{p}}:$ Mobilité des électrons et des trous.

$v \quad$ :Fréquence (généralement optique).

$\rho \quad:$ Rendement quantique interne.

$\varepsilon \quad$ : Constante diélectrique du silicium.

$\sigma \quad:$ Sensibilité d'un photodétecteur.

$\sigma_{0} \quad$ : Sensibilité d'une photodiode à avalanche pour $M=1$.

1. Introduction. - Le développement, dans le domaine des télécommunications par fibre optique, de sources de lumière cohérente (laser) ou incohérente (diodes électroluminescentes) pour véhiculer l'information, est allé de pair avec celui des photodétecteurs rapides et sensibles. Les photodiodes PIN permettent d'obtenir des temps de réponse inférieurs à la nanoseconde, mais pour leur utilisation en détection de signaux de faible amplitude, il faudrait leur associer des amplificateurs dont le produit gain-bande passante est nécessairement limité. Par contre, les photodiodes à avalanche semblent présenter un meilleur compromis entre l'amplification et la rapidité. S'inspirant de la diode de Read [1], Ruegg [2] proposa en 1967 une structure $\mathrm{N}^{+} \mathrm{P} \pi \mathrm{P}^{+}$qui possède les avantages du gain interne des photodiodes $\mathrm{N}^{+} \mathrm{P}$ à avalanche et ceux des photodiodes PIN : rapidité et sensibilité. Dans ces dispositifs à avalanche, il faut déterminer avec précision le facteur de multiplication qui intervient implicitement dans la détermination de la puissance

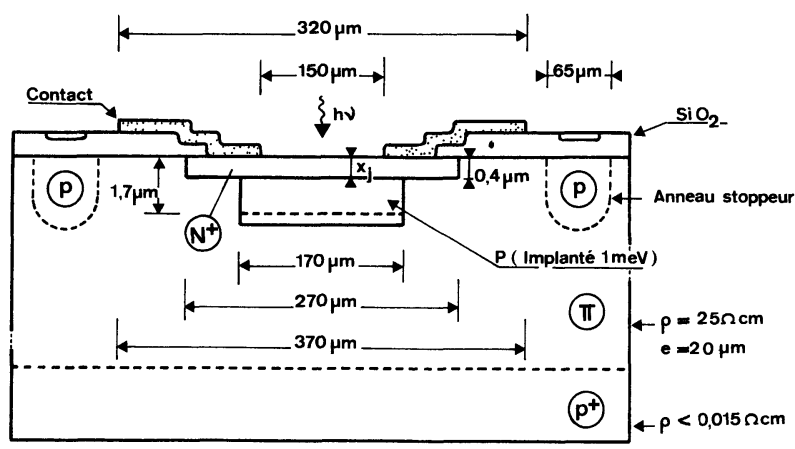

Fig. 1. - Vue en coupe d'une photodiode $\mathrm{N}^{+} \pi \mathrm{P} \pi \mathrm{P}^{+}$.

[Crossection of the $\mathrm{N}^{+} \pi \mathrm{P} \pi \mathrm{P}^{+}$diode.] équivalente de bruit. L'effet de l'extension de la zone de charge d'espace sur le photocourant primaire et par voie de conséquence sur le facteur de multiplication a été mis en évidence par V. W. Gartner [3]. En l'absence de toute multiplication par ionisation par impact, cet effet se traduit par une augmentation du photocourant. Dans la structure $\mathrm{N}^{+} \mathrm{P} \pi \mathrm{P}^{+}$lorsque les porteurs sont créés dans la zone $\pi$ et que la multiplication a lieu, il est alors impossible par des mesures au premier ordre d'atteindre le photocourant non multiplié. L'objet de ce papier est de déterminer ce paramètre à partir des mesures de bruit.

2. Description du dispositif. - Les photodiodes à avalanche étudiées (type CG 5700) sont fabriquées par les Laboratoires de Marcoussis, Centre de Recherches de la Compagnie Générale d'Electricité [4]. Ces composants ont été réalisés selon une géométrie circulaire sur des substrats épitaxiés de type $p$ de résistivité de l'ordre de 25 à $29 \Omega$.cm

$$
\left(N_{\pi} \simeq 5 \times 10^{1+} \mathrm{cm}^{3}\right) \text {. }
$$

La surface active a un diamètre de $150 \mu \mathrm{m}$. La figure 1 montre une vue en coupe du dispositif. La zone $p$ est obtenue par implantation ionique (énergie d'implantation $1 \mathrm{MeV}$ ) réalisée au CNET Lannion. Avec un tel profil d'impuretés la multiplication par ionisation par impact se produira au voisinage de la jonction $\mathrm{N}^{+} \mathrm{P}$. La jonction $\mathrm{N}^{+} \pi$ joue le rôle d'anneau de garde. L'anneau stoppeur a pour but de bloquer le courant de fuite de surface dû à la présence d'une couche d'inversion à l'interface $\mathrm{SiO}_{2}-\mathrm{Si}$. La profondeur de la jonction $x_{j}$ est voisine de $0,4 \mu \mathrm{m}$. Pour un fonctionnement correct de cette structure, la zone de charge d'espace de la jonction $\mathrm{N}^{+} \mathbf{P}$ doit atteindre la région $\pi$ pour une valeur finie $M_{0}$ de la multiplication. Une augmentation de la tension de polarisation au-delà de cette valeur entraînera l'apparition d'un palier dans la caractéristique courant-tension. La tension correspondant au début de ce palier est appelée tension de reach-through [2].

Pour une longueur d'onde donnée l'absorption et la création de photoporteurs se font plus ou moins profondément. La sensibilité maximum serait donc atteinte pour une photodiode assez longue. Ceci présente néanmoins, quelques inconvénients, à savoir :

- la limitation en fréquence à cause du temps de transit;

- la nécessité de polariser à très forte tension pour dépeupler complètement la zone $\pi$.

Un bon compromis entre la rapidité de détection et la sensibilité semble être une longueur de l'ordre de la profondeur d'absorption 1/a. Les photodiodes étudiées ont une longueur de $20 \mu \mathrm{m}$ et une sensibilité maximum au voisinage de $\lambda=0,8 \mu \mathrm{m}$ (Fig. 2). Ceci présente un double avantage.

i) Les sources de lumière à l'état solide au voisinage de cette longueur d'onde sont nombreuses. 


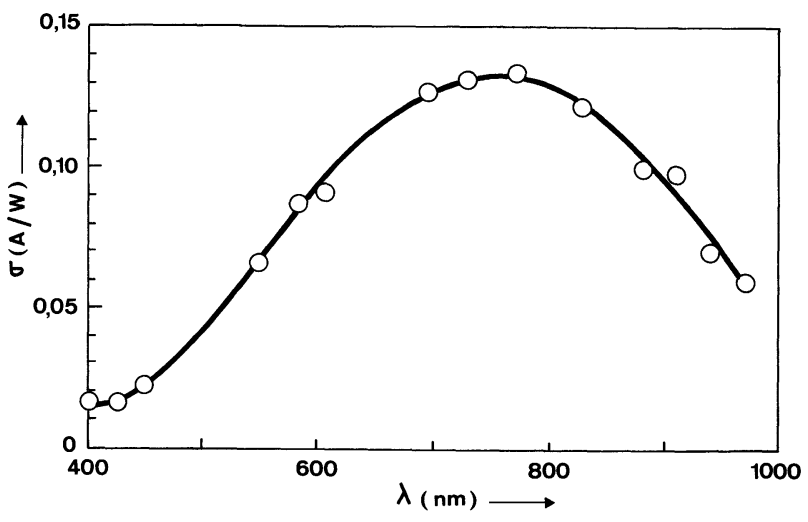

Fig. 2. - Sensibilité en $A / W$ en fonction de la longueur d'onde du dispositif $\mathrm{N}^{+} \pi \mathrm{P} \pi \mathrm{P}^{+}$.

[Sensitivity in $A / W$ as a function of wavelength of the $\mathrm{N}^{+} \pi \mathrm{P} \pi \mathrm{P}^{+}$ device.]

ii) La création de porteurs en profondeur dans ce type de structure entraîne une injection presque pure d'électrons qui sont les porteurs les plus ionisants dans le silicium.

Lorsque la zone $\pi$ est totalement dépeuplée, la rapidité de réponse des structures $\mathrm{N}^{+} \mathrm{P} \pi \mathrm{P}^{+}$est pratiquement semblable à celle des diodes PIN de même longueur, tout en bénéficiant des avantages du gain interne dû à la multiplication par ionisation par chocs.

3. Définition de différents paramètres intervenant dans le modèle de caractérisation de la photodiode à avalanche. - Considérons une structure $\mathrm{N}^{+} \mathrm{P} \pi \mathrm{P}^{+}$ (Fig. 3a). La concentration en impuretés dans la zone $\mathrm{N}^{+}$est suffisamment élevée pour supposer que la zone de charge d'espace s'étend uniquement dans la zone P. Celle-ci étant obtenue par implantation ionique, le profil de concentration est généralement assimilé à une distribution gaussienne (théorie de Lindhard, Scharff et Schiott $[5,6])$, dont la variation en fonction de la distance est donnée par la relation :

$$
|N(x)|=N_{\pi}+\frac{S}{2 \pi \Delta R_{\mathrm{p}}} \exp \left\{-\frac{\left(x-R_{\mathrm{p}}\right)^{2}}{2 \Delta R_{\mathrm{p}}^{2}}\right\}
$$

représentée sur la figure $3 b$.

La région de charge d'espace étant le siège d'un champ électrique intense, les porteurs y transiteront à leur vitesse limite. En supposant pratiquement toutes les impuretés ionisées, l'intégration de l'équation de Poisson compte tenu de la condition limite $\varepsilon(W)=0$ permet d'obtenir l'expression du champ électrique $\varepsilon(x)$ :

$$
\begin{aligned}
\varepsilon|(x)| & =\frac{q}{\varepsilon} N_{\pi}(W-x)+ \\
& +S\left|\operatorname{erf}\left(\frac{W-R_{\mathrm{p}}}{2 \Delta R_{\mathrm{p}}}\right)-\operatorname{erf}\left(\frac{x-R_{\mathrm{p}}}{2 \Delta R_{\mathrm{p}}}\right)\right|
\end{aligned}
$$

dont la variation en fonction de $x$ est représentée sur la figure $3 c$, le maximum étant obtenu en $x=0$.

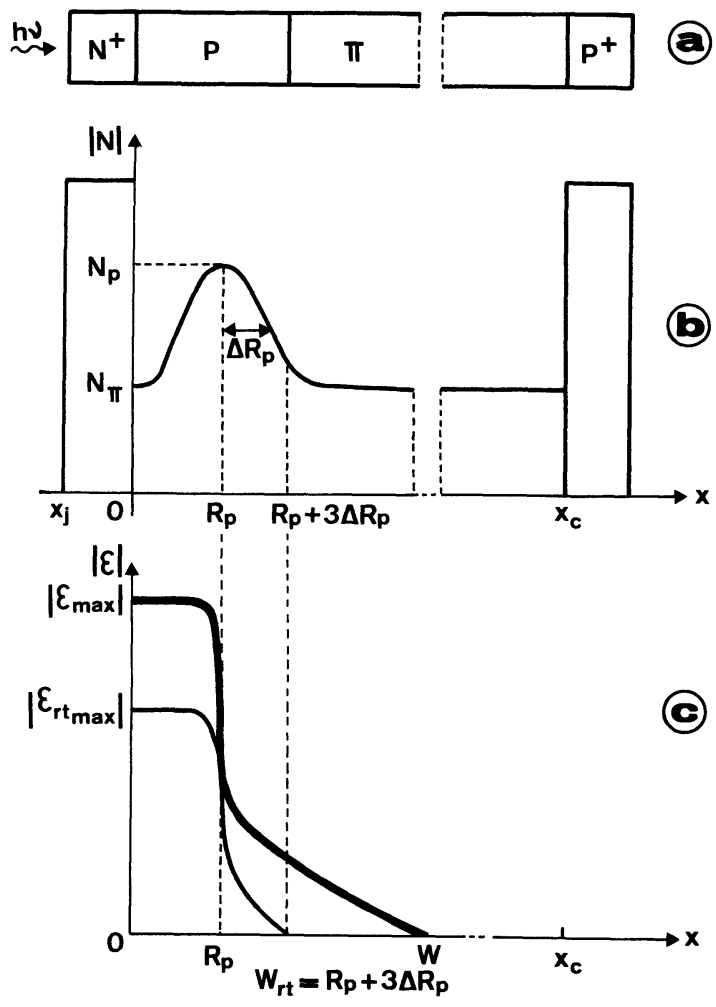

Fig. 3. - Profil de distribution des impuretés et champ électrique interne en fonction de $x$.

[Impurity profile and electric field as a function of $X$.]

Une seconde intégration nous donne la distribution $\mathrm{du}$ potentiel $V(x)$. Compte tenu de l'expression du champ électrique $\varepsilon(x)$ et de celle de $V(x)$, il est aisé de constater que l'influence de la zone $\mathrm{p}$ est directement liée à sa position dans la zone $\pi$. En effet si $R_{\mathrm{p}}$ est très grand, nous retrouvons les relations d'une jonction $\mathrm{N}^{+} \pi$ abrupte. Dans le but de se rapprocher le plus possible du modèle idéal de Ruegg [2], les paramètres du dispositif, plus particulièrement $R_{\mathrm{p}}, \Delta R_{\mathrm{p}}$ et $S$, doivent être ajustés de telle sorte que le champ électrique entre 0 et $R_{\mathrm{p}}$ dépasse le champ critique d'ionisation en régime normal de fonctionnement et que la

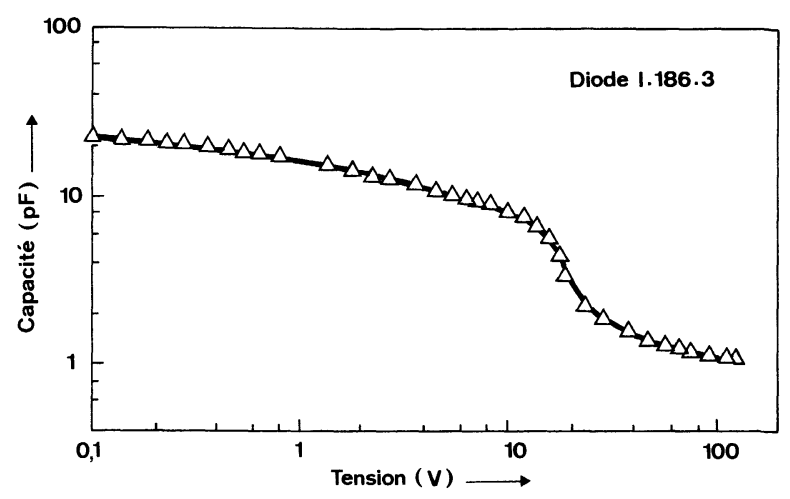

Fig. 4. - Variation de la capacité en fonction de la tension appliquée.

[The capacity as a function of the applied voltage.] 
zone $\pi$ soit entièrement dépeuplée. En analysant les variations de la capacité en fonction de la tension appliquée (Fig. 4) ainsi que la caractéristique $I=f(V)$ à l'obscurité (Fig. 5), on constate que la diode 1.186.3 étudiée se rapproche du modèle de Ruegg [2]. La tension $V_{\mathrm{rt}}$ déterminée sur la courbe $C=f(V)$ correspond tout à fait à celle déterminée sur la caractéristique $I=f(V)$. Nous n'allons pas ici analyser le courant d'obscurité qui dans le cas de notre échantillon varie entre $10^{-12} \mathrm{~A}$ et $4 \times 10^{-11} \mathrm{~A}$. En effet, cette quantité sera négligeable devant les photocourants toujours supérieurs à $10^{-8} \mathrm{~A}$.

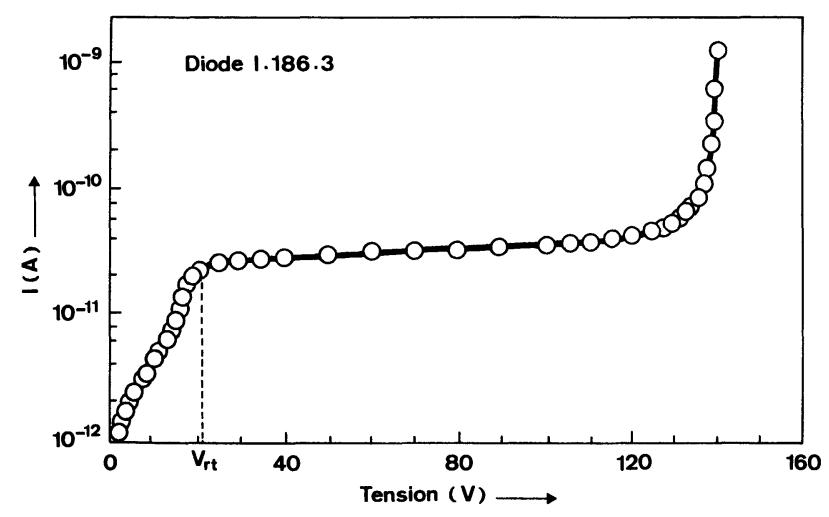

Fig. 5. - Caractéristique $I=f(V)$ à l'obscurité.

[Dark current plot $I=f(V)$.]

4. Expression analytique des photocourants primaires. - Soit $\varphi_{1}$ le flux de photons, d'énergie $h v$, arrivant sous incidence normale à la surface d'un dispositif d'épaisseur suffisante. Le flux de photons $\varphi_{0}=(1-R) \varphi_{1}$ pénétrant dans le cristal $y$ est donc entièrement absorbé. Chaque photon absorbé n'est pas forcément actif : absorption par des porteurs libres, dispersion par les imperfections du réseau cristallin... En tenant compte du coefficient d'absorption, le taux de génération de paires électron-trou à une distance $X$ de la surface du cristal est :

$$
G(x)=-\rho \frac{\mathrm{d}}{\mathrm{d} x} \varphi(x)=\rho a \varphi_{0} \mathrm{e}^{-a x} .
$$

Après excitation optique, la concentration des porteurs libres dans le cristal, se trouve augmentée. Durant leur durée de vie, les photoporteurs se déplacent sous l'influence du champ externe, du gradient de concentration etc... Ainsi dans le cas de la photodiode, la nature du photocourant dépendra du processus de transport des photoporteurs. Dans la zone de charge d'espace, le courant est essentiellement un courant de conduction (en négligeant les recombinaisons), tandis que dans les zones neutres le courant est essentiellement celui dû à la diffusion des porteurs minoritaires. Nous ne considérons que le cas unidimensionnel. Pour obtenir la distribution des porteurs en l'absence d'ionisation par impact, il nous faut résoudre les trois équations suivantes :

a) L'équation de continuité qui tient compte de la génération et de la recombinaison des porteurs de charge en excès.

b) L'équation de transport qui décrit le mouvement des porteurs sous l'influence du champ électrique et de la diffusion.

c) L'équation de Poisson.

Les hypothèses simplificatrices sont les suivantes :

i) Génération à faible niveau, c'est-à-dire que la concentration des porteurs majoritaires reste pratiquement inchangée, tandis que celle des porteurs minoritaires peut être fortement modifiée.

ii) La recombinaison en volume est une fonction linéaire de la concentration des porteurs minoritaires en excès.

iii) La recombinaison en surface est directement proportionnelle à la concentration en excès à la surface.

Soit une jonction $\mathrm{N}^{+} \mathrm{P}$ soumise à une excitation optique du côté $\mathrm{N}^{+}$(Fig. 3a). Compte tenu de l'orientation des axes, le taux de génération de porteurs à l'intérieur du cristal est donné par :

$$
G(x)=\rho a \varphi_{0} \exp \left\{-a\left(x+x_{\mathrm{j}}\right)\right\} .
$$

Le niveau d'injection sera supposé assez faible de telle sorte que la concentration des porteurs majoritaires de part et d'autre de la jonction demeure inchangée. Le courant est donc régi par les porteurs minoritaires. De plus, dans l'hypothèse des courants faibles, le champ électrique est sensiblement nul à l'extérieur de la zone déplétée et la composante du courant de conduction est négligeable. Dans la zone de déplétion, en raison du champ électrique intense qui y règne, les porteurs sont séparés et participent à la conduction, on suppose qu'ils transitent à leur vitesse limite et que le temps de transit est nul.

$\mathrm{Du}$ fait de la valeur différente des coefficients d'ionisation des trous et des électrons dans le cas du silicium, on décompose le photocourant primaire suivant la nature des porteurs injectés dans la zone de multiplication.

4.1 Photocourant primaire de trous injectés DANS LA ZONE DE MULTIPLICATION. - Il est constitué par la diffusion des porteurs créés dans la zone $\mathrm{N}^{+}$. En intégrant l'équation de continuité (a) avec les hypothèses simplificatrices (i), on obtient en tenant compte des conditions aux limites suivantes :

$$
\begin{array}{lll}
\text { en } x=0 & \Delta p=0 \\
\text { en } & x=-x_{\mathrm{j}}, & \frac{\mathrm{d}}{\mathrm{d} x} \Delta p=+\frac{s}{D_{\mathrm{p}}} \Delta p
\end{array}
$$




$$
I_{\mathrm{p}_{0}}=q \rho \varphi_{0} A \mathrm{e}^{-a x_{\mathrm{j}}} \frac{a L_{\mathrm{p}}}{1-a^{2} L_{\mathrm{p}}^{2}}\left\{\frac{\frac{s}{D_{\mathrm{p}}} \cosh \left(\frac{x_{\mathrm{j}}}{L_{\mathrm{p}}}\right)+\frac{1}{L_{\mathrm{p}}} \sinh \left(\frac{x_{\mathrm{j}}}{L_{\mathrm{p}}}\right)-\left(a+\frac{s}{D_{\mathrm{p}}}\right) \mathrm{e}^{a x_{\mathrm{j}}}}{\frac{1}{L_{\mathrm{p}}} \cosh \left(\frac{x_{\mathrm{j}}}{L_{\mathrm{p}}}\right)+\frac{s}{D_{\mathrm{p}}} \sinh \left(\frac{x_{\mathrm{j}}}{L_{\mathrm{p}}}\right)}+a L_{\mathrm{p}}\right\} .
$$

Cette composante du photocourant primaire est indépendante de la tension appliquée et elle est surtout sensible à la profondeur de la jonction $x_{\mathbf{j}}$, à la vitesse de recombinaison en surface $s$ et au taux d'absorption $a$.

\subsection{Photocourant PRIMAIRE D'ÉLECTRONS INJEC-} TÉS DANS LA ZONE DE MULTIPLICATION. - Cette composante du photocourant est composée de deux parties :
- Courant de conduction des porteurs créés dans la partie de la zone de charge d'espace où il n'y a pas ionisation par impact.

- Courant de diffusion des porteurs créés à l'extérieur de la région de charge d'espace.

De la même manière que dans le paragraphe 4.1 on obtient l'expression du photocourant :

$$
I_{\mathrm{N}_{0}}=q \rho \varphi_{0} A \mathrm{e}^{-a x_{\mathrm{j}}}\left\{\mathrm{e}^{-a W_{\mathrm{A}}}-\mathrm{e}^{-a W}-\frac{a L_{\mathrm{n}}^{2}}{1-a^{2} L_{\mathrm{n}}^{2}} \mathrm{e}^{-a W}\left[a+\frac{\mathrm{e}^{-a\left(x_{\mathrm{c}}-W\right)}-\cosh \left(\frac{x_{\mathrm{c}}-W}{L_{\mathrm{n}}}\right)}{L_{\mathrm{n}} \sinh \left(\frac{x_{\mathrm{c}}-W}{L_{\mathrm{n}}}\right)}\right]\right\}
$$

avec les conditions aux limites suivantes :

$$
\text { en } \begin{aligned}
x & =W & \Delta n & =0 \\
x & =x_{\mathrm{c}} & \Delta n & =0 .
\end{aligned}
$$

On constate que cette composante du photocourant primaire dépend par l'intermédiaire de $W$ de la tension de polarisation. D'autre part, le dernier terme s'annule quand $x_{\mathrm{c}}=W$ et $I_{\mathrm{N}_{0}}$ se sature du fait que $W$ ne peut plus s'étendre au-delà de $x_{\mathrm{c}}$. On obtient donc pour la composante maximale $I_{\mathrm{N}_{0} \text { sat }}$ l'expression :

$$
I_{\mathrm{N}_{0} \text { sat }}=q \rho \varphi_{0} A \mathrm{e}^{-a x_{\mathrm{j}}}\left(\mathrm{e}^{-a W_{\mathrm{A}}}-\mathrm{e}^{-a x_{\mathrm{c}}}\right) .
$$

4.3 PhOtocourant PRIMAIRE DES PORTEURS CRÉÉS DANS LA ZONE DE MULTIPLICATION. - Cette composante du photocourant est due aux porteurs créés uniquement dans la zone de multiplication, soit :

$$
J_{W_{\mathrm{a}}}=q A \int_{0}^{W_{\mathrm{A}}} \rho a \varphi_{0} \mathrm{e}^{-a\left(x+x_{\mathrm{j}}\right)} \mathrm{d} x
$$

ce qui donne en intégrant :

$$
J_{W_{\mathbf{A}}}=q \rho \varphi_{0} A \mathrm{e}^{-a x_{\mathbf{j}}}\left(1-\mathrm{e}^{-a W_{\mathbf{A}}}\right) .
$$

4.4 Photocourant PRimaire total. - Le photocourant primaire total qui traverse la diode en l'absence de multiplication est la somme des trois relations (7), (8) et (12). De ces trois composantes c'est la relation (8) qui montre la dépendance du photocourant primaire avec la tension de polarisation appliquée par l'intermédiaire de $W$ qui n'est rien d'autre que l'extension de la zone de charge d'espace.
En présence de multiplication, cet effet entraîne une indétermination du facteur de multiplication $M$. En effet, la variation du courant total est consécutive à la conjugaison des deux processus. Pour ce type de structure $\mathrm{N}^{+} \mathrm{P} \pi \mathrm{P}^{+}$, il serait néfaste de réduire cette composante du photocourant donnée par la relation (8) car d'une part la multiplication par les électrons est plus grande que celle due aux trous et d'autre part $\mathrm{du}$ point de vue du bruit, l'injection des porteurs les plus ionisants (les électrons dans le $\mathrm{Si}$ ) est la moins bruyante.

5. Calcul de la variation du photocourant primaire en fonction de la tension de polarisation en l'absence de multiplication. - Nous allons calculer l'expression du photocourant total en l'absence de multiplication pour deux structures $\mathrm{N}^{+} \pi \mathbf{P}^{+}$et $\mathbf{N}^{+} \mathbf{P} \pi \mathbf{P}^{+}$éclairées du côté $\mathrm{N}^{+}$à différentes longueurs d'onde c'est-à-dire pour différents coefficients d'absorption optique $a$ correspondant à des créations de photoporteurs en profondeur et en surface. Si les photoporteurs sont créés en surface le photocourant doit être indépendant de la tension appliquée. Par contre, dans le cas de création en profondeur, l'effet de l'extension de la zone de charge d'espace doit être sensible et le photocourant doit varier en fonction de la tension appliquée. La figure 6 représente la variation du rapport calculé de $I_{\mathrm{ph}}(V) / I_{\mathrm{ph}}(0)$ en fonction de la tension appliquée pour la structure $\mathrm{N}^{+} \pi \mathrm{P}^{+}$. Pour un coefficient d'absorption $a=5 \times 10^{4} \mathrm{~cm}^{-1}$ correspondant à une longueur d'onde $\lambda$ voisine de $426 \mathrm{~nm}$, tous les porteurs sont créés en surface et le rapport $I_{\mathrm{ph}}(V) / I_{\mathrm{ph}}(0)$ reste égal à 1 . Pour les autres coefficients d'absorption c'est-à- 


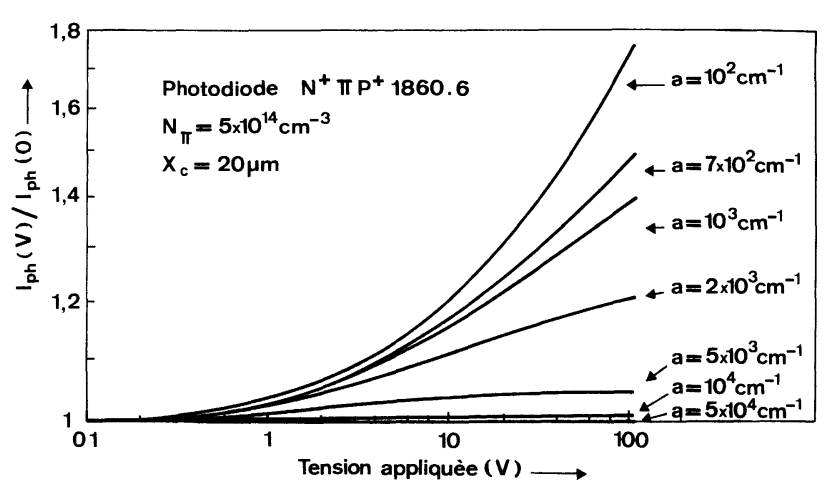

Fig. 6. - Variation calculée du rapport $I_{\mathrm{ph}}(V) / I_{\mathrm{ph}}(0)$ en fonction de la tension appliquée pour différents coefficients d'absorption pour la diode $\mathrm{N}^{+} \pi \mathrm{P}^{+}$.

[Calculated ratio $I_{\mathrm{ph}}(V) / I_{\mathrm{ph}}(0)$ as a function of the applied voltage for different absorption coefficients of the $\mathrm{N}^{+} \pi \mathrm{P}^{+}$diode.]

dire pour des créations de photoporteurs en profondeur, on voit que l'extension de la zone de charge d'espace entraîne une variation du photocourant en fonction de $V$. Cette variation peut atteindre jusqu'à $80 \%$ dans l'infrarouge. On peut vérifier expérimentalement ce résultat en mesurant la photocaractéristique $I_{\mathrm{ph}}=f(V)$ d'une structure $\mathrm{N}^{+} \pi \mathrm{P}^{+}$aux longueurs d'onde $\lambda=426 \mathrm{~nm}$ et $\lambda=827 \mathrm{~nm}$. Ces courbes sont représentées sur la figure 7. A $\lambda=426 \mathrm{~nm}$ la création des photoporteurs se fait en surface et sa valeur sera indépendante de l'extension de la zone de charge d'espace dans la zone $\pi$ donc de la tension $V$ appliquée. On constate en effet sur la figure 7 que le photocourant reste égal à $10^{-8} \mathrm{~A}$ jusqu'au seuil de la tension de claquage. Résultat qui se trouve en bon accord avec celui calculé et représenté sur la figure 6 . Par contre, à $\lambda=827 \mathrm{~nm}$ pour laquelle les photoporteurs sont créés en profondeur dans la zone $\pi$ nous observons qu'entre $0 \mathrm{~V}$ et $170 \mathrm{~V}$, le photocourant primaire de $10^{-8} \mathrm{~A}$ a varié d'un facteur 1,9

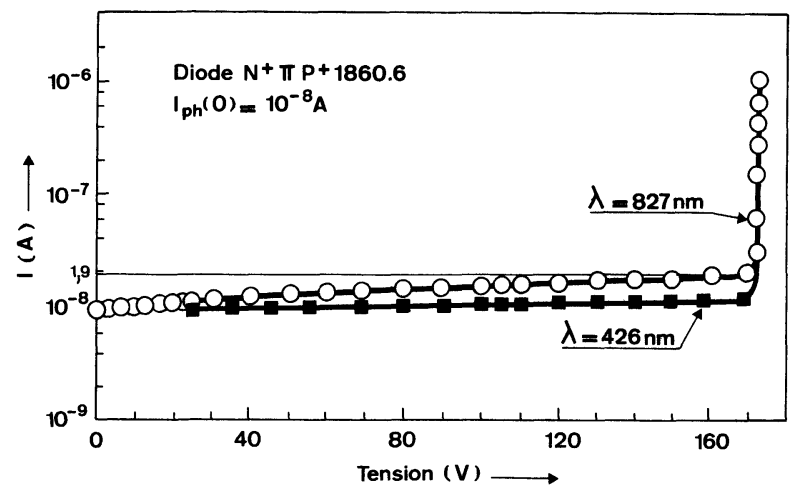

Fig. 7. - Caractéristique $I=f(V)$ de la diode $\mathrm{N}^{+} \pi \mathrm{P}^{+}$à deux longueurs d'onde correspondant à la création des photoporteurs en surface $(\lambda=426 \mathrm{~nm})$ et en profondeur $(\lambda=827 \mathrm{~nm})$.

[Current-voltage plot of the $\mathrm{N}^{+} \pi \mathrm{P}^{+}$diode at two wavelengths corresponding to a creation of photocarriers at the surface $(\lambda=426 \mathrm{~nm})$ and at a greater depth $(\gamma=827 \mathrm{~nm})$. en bon accord avec le résultat calculé sur la figure 6 . Cette augmentation du photocourant dans ce cas d'expérience est due, en l'absence de toute multiplication à l'extension de la zone de charge d'espace.

Pour la structure $\mathrm{N}^{+} \mathrm{P} \pi \mathrm{P}^{+}$, cette vérification expérimentale ne sera pas possible du fait de la présence simultanée de l'ionisation par impact et de l'extension de la zone de charge d'espace. Néanmoins, on peut toujours calculer l'expression analytique du rapport du photocourant $I_{\mathrm{ph}}(V) / I_{\mathrm{ph}}(0)$ en fonction de la tension de polarisation $V$; ceci est représenté sur la figure 8 pour différents coefficients d'absorption. Si les porteurs sont créés en surface $\left(a=5 \times 10^{4} \mathrm{~cm}^{-1}\right)$ le photocourant primaire est indépendant de la tension appliquée. Par contre, lorsque les photoporteurs sont créés de plus en plus loin, c'est-à-dire dans la zone $\pi$, nous voyons apparaître l'influence de l'extension de la zone de charge d'espace qui devient prépondérante dès que la tension appliquée dépasse la tension de reach through. Pour $a=10^{2} \mathrm{~cm}^{-1}$ l'écart peut atteindre jusqu'à $80 \%$.

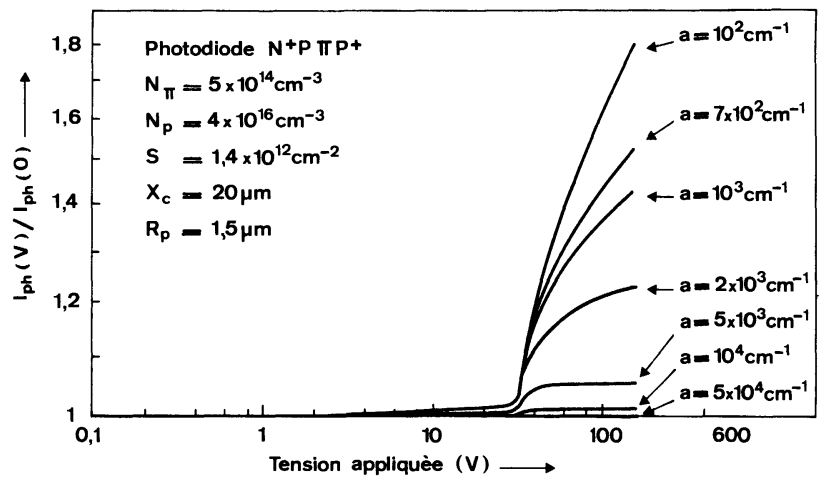

Fig. 8. - Variation calculée du rapport $I_{\mathrm{ph}}(V) / I_{\mathrm{ph}}(0)$ en l'absence de multiplication pour la diode $\mathrm{N}^{+} \mathrm{P} \pi \mathrm{P}^{+}$.

[Calculated ratio $I_{\mathrm{ph}}(V) / I_{\mathrm{ph}}(0)$ without any multiplication for the diode $\mathrm{N}^{+} \mathrm{P} \pi \mathrm{P}^{+}$.]

On définit habituellement le coefficient de multiplication $M$ comme étant le rapport du photocourant $I_{\mathrm{ph}}(V)$ à la tension $V$ sur le photocourant $I_{\mathrm{ph}}(0)$ à la tension $0 \mathrm{~V}$ ou à une tension faible devant la tension de claquage. Cette définition, exacte pour une création en surface des photoporteurs entraîne une erreur importante dans le cas d'une création en profondeur dans la zone $\pi$. En effet, le vrai photocourant primaire multiplié n'est pas celui défini à $0 \mathrm{~V}$, mais celui qui existe vraiment à la tension d'utilisation qui tiendra compte de l'extension de la zone de charge d'espace. Si toute la zone $\pi$ est dépeuplée alors le vrai courant primaire multiplié est celui défini par la relation (10) c'est-à-dire le $I_{\mathrm{N}_{0} \text { sat }}$. Ne pouvant pas atteindre ce paramètre sur les caractéristiques $I_{\mathrm{ph}}(V)=f(V)$ (Fig. 9), nous utilisons alors la caractérisation en bruit de ce type de dispositif. 


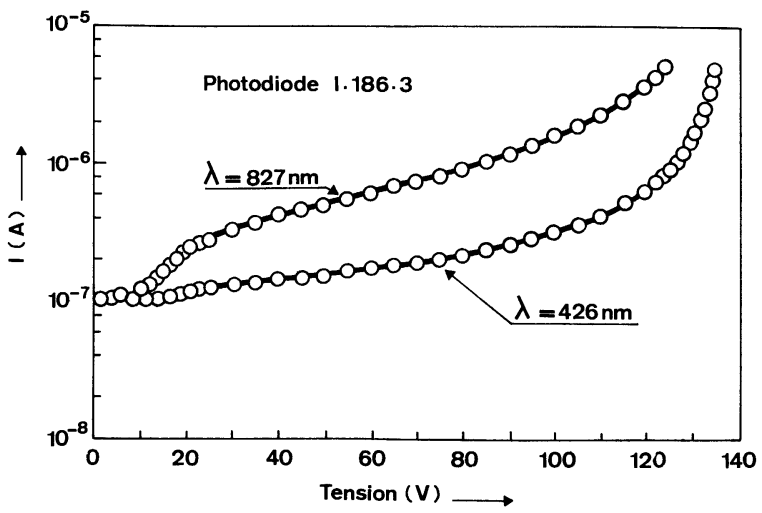

Fig. 9. - Caractéristique $I=f(V)$ à deux longueurs d'onde correspondant à la création des photoporteurs en surface $(\lambda=426 \mathrm{~nm})$ et en profondeur $(\lambda=827 \mathrm{~nm})$.

[Current-voltage plot of the $\mathrm{N}^{+} \mathrm{P} \pi \mathrm{P}^{+}$diode at two wavelengths corresponding to a creation of photocarriers at the surface $(\lambda=426 \mathrm{~nm})$ and at a greater depth $(\lambda=827 \mathrm{~nm})$.

6. Bruit dans les photodiodes à avalanche $\mathbf{N}^{+} \mathbf{P} \pi \mathbf{P}^{+}$. - Les photodiodes à avalanche sont uniquement utilisées en régime de multiplication. On a d'après la théorie de McIntyre [7] les relations suivantes :

- Pour une injection pure de trous :

$S_{\mathrm{i}}(f)=2 q I_{\mathrm{inj}} M_{\mathrm{p}}^{3}\left|1+\frac{1-k}{k}\left(\frac{M_{\mathrm{p}}-1}{M_{\mathrm{p}}}\right)^{2}\right|$.

- Pour une injection pure d'électrons :

$S_{\mathrm{i}}(f)=2 q I_{\mathrm{inj}} M_{\mathrm{n}}^{3}\left|1-(1-k)\left(\frac{M_{\mathrm{n}}-1}{M_{\mathrm{n}}}\right)^{2}\right|$.

Expérimentalement le bruit associé aux photodiodes à avalanche peut être approximé par l'expression :

$$
S_{\mathrm{i}}(f)=2 q I_{\mathrm{inj}} M^{x} \text { avec } 2<x<4
$$

avec $x=3$ quand $\alpha=\beta$.

Si $I_{\text {inj }}$ est le photocourant $I_{0}(0)$ déterminé à $0 \mathrm{~V}$ et $I$ le courant total qui traverse la diode à la tension $V$, l'expression (15) peut encore s'écrire compte tenu du fait que $M=I / I_{0}(0)$.

$$
S_{\mathrm{i}}(f)=2 q I_{0}^{1-x} \cdot I^{x} .
$$

Deux cas peuvent se présenter :

a) Le courant primaire $I_{0}$ est indépendant de la tension de polarisation. La courbe représentative de $S_{\mathrm{i}}(f)$ en fonction du courant de polarisation $I$ de la diode est une droite $D$ de pente $x$ en coordonnées logarithmiques.

b) Le courant primaire $I_{0}$ est une fonction de la tension de polarisation. Les points figuratifs de $S_{\mathrm{i}}(f)$ pour chaque valeur de $I$, se trouveront sur une famille de droites parallèles $D_{\mathrm{i}}$ dont l'intersection avec $D_{0}=2 q I$ détermine le courant primaire.

Nous simulons ce résultat sur la figure 10, qui représente la variation théorique de $S_{\mathrm{i}}(f)$ en fonction du courant $I$ de polarisation de la diode en coordonnées logarithmiques. Il apparaît sur cette figure que si la multiplication n'a pas eu lieu, mais que le courant primaire $I_{0}$ varie avec la tension, on obtiendrait du bruit de grenaille pur, $S_{\mathrm{i}}(f)=2 q I$ (section $\mathrm{AB}$ ). Par contre, en présence de multiplication, le point figuratif de $S_{\mathrm{i}}(f)$ pour un courant $I_{\mathrm{m}}$ donné, correspondant à un courant primaire $I_{0 \mathrm{~m}}$ se situe sur la droite $D_{\mathrm{m}}=2 q I_{0 \mathrm{~m}}^{1-x} I^{x}$ avec $I=I_{\mathrm{m}}$ (point $\mathrm{C}$ ). Si nous ne tenions pas compte de la variation du courant primaire en fonction de la polarisation, dans la définition du coefficient de multiplication, le point figuratif se trouverait en $E$ [8].

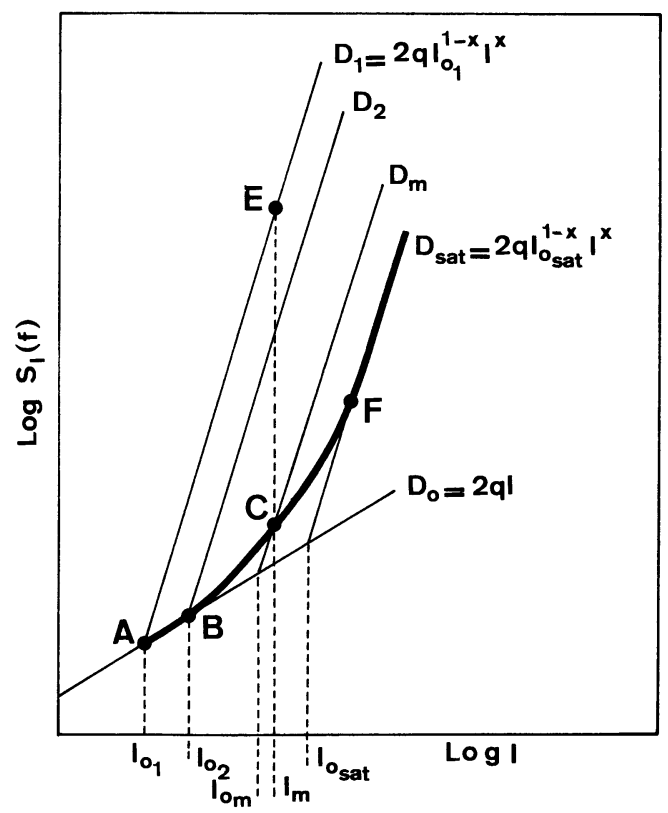

Fig. 10. - Variations calculées de $S_{\mathrm{i}}(f)$ en fonction du courant de polarisation compte tenu de l'extension de la zone de charge d'espace de la diode $\mathrm{N}^{+} \pi \mathrm{P} \pi \mathrm{P}^{+}$.

[The calculated current spectral density as a function of the current, taking into account the extension of the space charge region of the diode $\mathrm{N}^{+} \pi \mathrm{P} \pi \mathrm{P}^{+}$.]

Lorsque le courant primaire se sature $\left(I_{0 \text { sat }}\right)$, c'est-à-dire que toute la région $\pi$ est déplétée jusqu'au $\mathrm{P}^{+}\left(W=x_{\mathrm{c}}\right)$ (Figs $3 a$ et $\left.3 b\right)$, le bruit est représenté par la droite $D_{\text {sat }}=2 q I_{0 \text { sat }}^{1-x} I^{x}$.

La détermination précise du courant primaire et par voie de conséquence du coefficient de multiplication revêt donc une importance primordiale dans l'évaluation de la grandeur du bruit associé à un processus de multiplication. Le rapport entre la valeur du bruit si on prend comme courant primaire, celui à $0 \mathrm{~V} I_{0}^{\prime}$ et la valeur réelle $I_{0}$ est donné par :

$$
\frac{2 q I_{0}^{1-x} I^{x}}{2 q I_{0}^{1-x} I^{x}}=\left(\frac{I_{0}^{\prime}}{I_{0}}\right)^{1-x}=\left(\frac{I_{0}}{I_{0}^{\prime}}\right)^{x-1} .
$$

Si $\left(I / I_{0}\right)$ est de 1,8 et $x=2,5$ le rapport est de 2,41 $(\simeq 4 \mathrm{~dB})$. 
Sur la figure 11, nous avons reporté la courbe de bruit expérimentale pour une photodiode de structure $\mathrm{N}^{+} \mathrm{P} \pi \mathrm{P}^{+}$(I.186.3) à deux longueurs d'onde différentes $(\lambda=426 \mathrm{~nm}$ et $\lambda=827 \mathrm{~nm})$. Nous constatons que pour une création de porteurs en surface $(\lambda=426 \mathrm{~nm})$ le photocourant primaire n'est pas affecté par l'extension de la zone de charge d'espace et demeure pratiquement constant. $S_{\mathrm{i}}(f)=f(I)$ est représenté par une droite de pente 3,93 qui démarre à partir de $I_{\mathrm{ph}}(0 \mathrm{~V})=10^{-7} \mathrm{~A}$. Par contre, pour une création en profondeur $(\lambda=827 \mathrm{~nm})$ et la même valeur du courant primaire à $0 \mathrm{~V}\left(10^{-7} \mathrm{~A}\right)$, le compor-

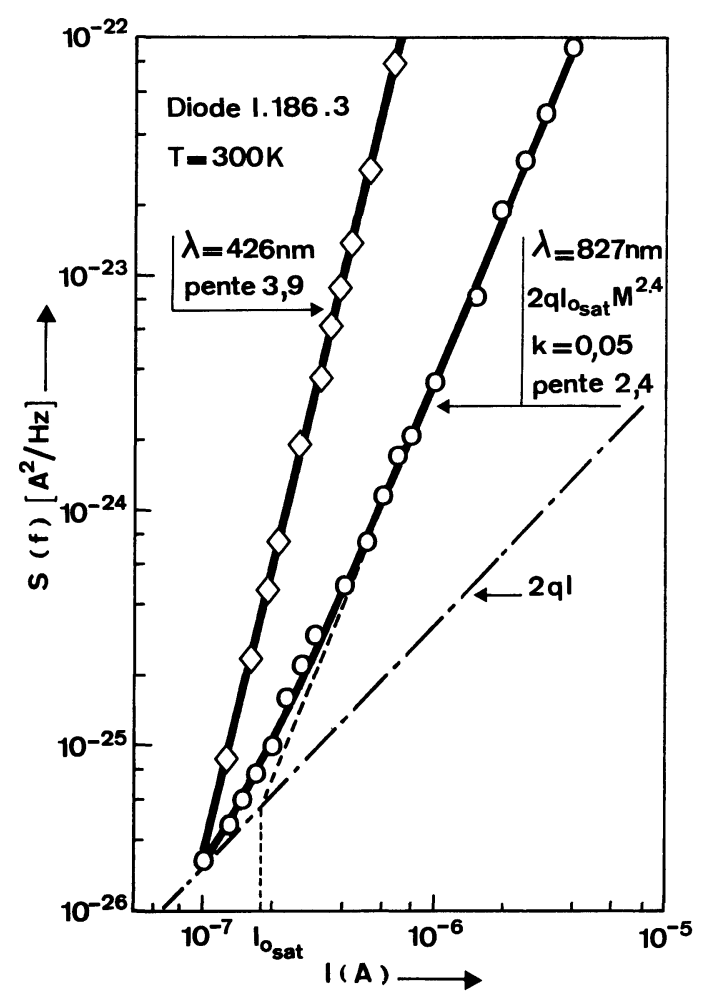

Fig. 11. - Densité spectrale $S_{\mathrm{i}}(f)$ en fonction du courant de polarisation à $\lambda=426 \mathrm{~nm}$ et à $\lambda=827 \mathrm{~nm}$.

[Current spectral density as a function of the current at $\lambda=426 \mathrm{~nm}$ and $\lambda=827 \mathrm{~nm}$. tement du bruit est semblable à celui simulé sur la figure 10. L'intersection de la droite $2 q I_{0 \text { sat }} M^{2,4}$ avec la droite $2 q I$ (Fig. 11), nous donne la valeur du courant primaire $I_{0 \text { sat }}$ qui est injecté dans la zone de multiplication lorsque toute la zone $\pi$ est déplétée. Compte tenu de la précision des mesures de bruit $(\simeq 10 \%)$ le rapport du courant primaire à la saturation sur celui mesuré à la tension nulle, peut être estimé à 2 . L'influence sur le facteur de multiplication est directe puisque si l'on s'en tient à la définition classique, on obtiendrait un facteur de multiplication $M$ deux fois plus grand que celui existant réellement.

Notons que ce phénomène n'est pas propre aux structures $\mathrm{N}^{+} \mathrm{P} \pi \mathrm{P}^{+}$mais valable pour tous les photodétecteurs à zone de déplétion comme l'a montré Gärtner [3]. Seulement l'effet n'est sensible que pour les dispositifs à forte résistivité. En ce qui nous concerne, la caractérisation par la méthode de mesure de bruit de photodiodes à avalanche nous permet donc de lever l'ambiguité quant à la détermination expérimentale du facteur de multiplication qui au premier ordre est impossible à réaliser du fait que l'on ne peut pas dissocier le photocourant primaire du courant photoélectrique total.

Ainsi, pour une photodiode, le facteur de multiplication $M$ peut s'exprimer à partir de la relation (16), connaissant les grandeurs expérimentales du bruit, du courant de polarisation $I$ et du coefficient $x$, par :

$$
\frac{S_{\mathrm{i}}(f)}{2 q I}=I_{0}^{1-x} I^{x-1}=M^{x-1}
$$

d'où

$$
M=\left(\frac{S_{\mathrm{i}}(f)}{2 q I}\right)^{1 /(x-1)}
$$

7. Conclusion. - L'indétermination du facteur de multiplication $M$ due à l'extension de la zone de charge d'espace est levée par l'étude en bruit de ce dispositif. Il est en effet important de connaître assez précisément les valeurs de $M$ si l'on veut utiliser de façon optimum ce photodétecteur pour une application donnée.

\section{Bibliographie}

[1] Read, W. T., Bell. Syst. Tech. J. 37 (1958) 401.

[2] RuEgG, H. W., I.E.E.E. Trans. on Electron. Devices ED-14 (1967) 239.

[3] Gartner, W. W., Phys. Rev. 116 (1959) 84.

[4] Benoit, J., Ripoche, G., Contrat D.G.R.S.T. $\mathrm{n}^{\mathrm{0}}$ 72.7.0518.00.221.75.01 (1973).
[5] Mayer, J. W., Eriksson, L., Davies, J. A., Ion implantation in Semiconductors (Acad. Press) 1970.

[6] Lecrosnier, D. et al., Techn. Dig. Int. Electron. Devices Meeting, pp. 595-597, Washington D.C. (1975).

[7] MC InTYRe, I.E.E.E. Trans. Electron. Devices ED 13 (1966) 164.

[8] RatsiRa, D., Thèse de 3e cycle, Montpellier (1978). 